\title{
MicroscopyPioneers
}

\section{Pioneers in Optics: Jacques Babinet}

\author{
Michael W. Davidson
}

National High Magnetic Field Laboratory, Florida State University, Tallahassee, FL 32306

davidson@magnet.fsu.edu

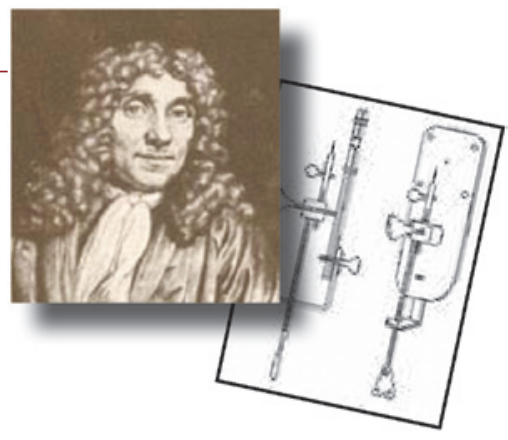

\section{Jacques Babinet}

(1794-1872)

Jacques Babinet was a French physicist, mathematician, and astronomer born in Lusignan, who is most famous for his contributions to optics. Among Babinet's accomplishments are the 1827 standardization of the Ångström unit for measuring light using the red cadmium line's wavelength, and the principle (bearing his name) that similar diffraction patterns are produced by two complementary screens.

After initiating his studies at the Lycée Napoléon, Babinet was persuaded to abandon his legal education for the pursuit of science. Formally educated at the École Polytechnique, which he left in 1812 for the Military School at Metz, Babinet was later a professor at the Sorbonne, and in 1840 he was elected a member

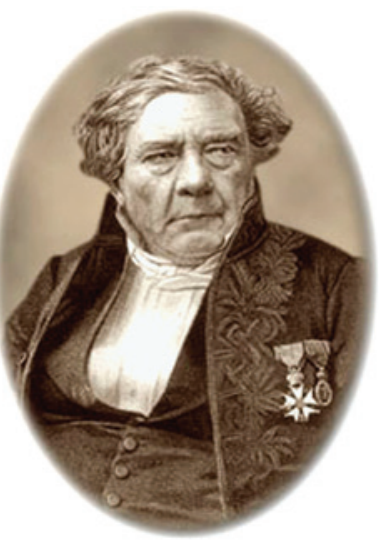
of the French Academy of Sciences. In addition to his brilliant lectures on meteorology and optics research, Babinet was also a great promoter of science, an amusing and clever lecturer, and a brilliant and entertaining author of popular scientific articles. Unlike many of his contemporaries, Babinet was beloved by all for his kindly and charitable nature.

Babinet was interested in the optical properties of minerals throughout his career. He designed and created many scientific instruments used to determine crystalline structure and polarization properties, including the polariscope and a goniometer to measure refractive indices. The Babinet compensator, an accessory useful in polarized light microscopy, was built with twin, opposed quartz wedges having mutually perpendicular crystallographic axes, and it is still widely employed in microscopy. This design avoids the problems inherent in the basic quartz wedge, where the zero reading coincides with the thin end of the wedge, which is often lost when grinding the plate during manufacture.
Expanding his fascination of diffraction to meteorology, Babinet spent a significant amount of time in the study of rainbow optics. His astronomical research focused on Mercury's mass and the Earth's magnetism, while his inventions included valve improvements for air pumps and a hygrometer. In geography and hydrogeomorphology, the Baer-Babinet Law helps to explain and predict directionality in the course of rivers. Babinet's cartography work includes homalographic projections where the parallels are rectilinear and meridian lines are elliptical.

The Babinet compensator is an optical device that enables the addition of a controllable retardation to the optical path difference of a specimen in polarized light microscopy. Unlike a fixed wave plate where the path difference is fixed, a

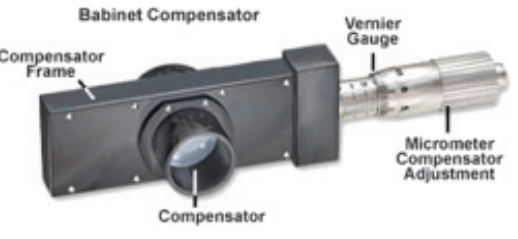
Babinet compensator can be adjusted to provide a variable path difference.

The Babinet compensator consists of paired quartz wedges, which are cut in such a fashion that one is positioned with the optical axis parallel to the edge, while the other has the axis perpendicular to the edge. The optical path difference in each wedge increases from the edge to the base, and the birefringence has opposite values in the wedges. When combined, a line appears where the net optical path difference through the compensator becomes zero, and increases in a direction at right angles to the zero line as the distance grows larger. Between crossed polarizers, dark bands are observed in monochromatic light at a separation distance of one wavelength of optical path difference. In white light, the polarization colors appear in rising orders. In polarized light microscopy, measurements are conducted by observing the fringe shift caused by the addition of the optical path difference of the specimen under observation.

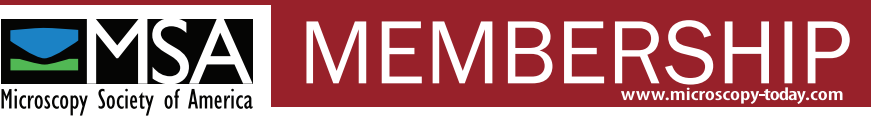

